Evaluation of optical imaging and spectroscopy approaches for cardiac tissue depth assessment

B. Lin, D. Matthews, V. Chernomordik, A. Gandjbakhche, S. Lane, S. G. Demos

February 21, 2008

Photonics West 2008

San Jose, CA, United States

January 18, 2008 through January 24, 2008 
This document was prepared as an account of work sponsored by an agency of the United States government. Neither the United States government nor Lawrence Livermore National Security, LLC, nor any of their employees makes any warranty, expressed or implied, or assumes any legal liability or responsibility for the accuracy, completeness, or usefulness of any information, apparatus, product, or process disclosed, or represents that its use would not infringe privately owned rights. Reference herein to any specific commercial product, process, or service by trade name, trademark, manufacturer, or otherwise does not necessarily constitute or imply its endorsement, recommendation, or favoring by the United States government or Lawrence Livermore National Security, LLC. The views and opinions of authors expressed herein do not necessarily state or reflect those of the United States government or Lawrence Livermore National Security, LLC, and shall not be used for advertising or product endorsement purposes. 


\title{
Evaluation of optical imaging and spectroscopy approaches for cardiac tissue depth assessment
}

\author{
Bevin Lin ${ }^{1}$, Dennis Matthews ${ }^{1,2}$, Victor Chernomordik ${ }^{3}$, Amir Gandjbakhche $^{3}$, Steve Lane ${ }^{1,2}$, and \\ Stavros Demos ${ }^{1,2}$ \\ University of California at Davis, Center for Biophotonics Science and Technology ${ }^{1}$ \\ Lawrence Livermore National Laboratory ${ }^{2}$ \\ National Institute of Health ${ }^{3}$
}

\begin{abstract}
NIR light scattering from ex vivo porcine cardiac tissue was investigated to understand how imaging or point measurement approaches may assist development of methods for tissue depth assessment. Our results indicate an increase of average image intensity as thickness increases up to approximately $2 \mathrm{~mm}$. In a dual fiber spectroscopy configuration, sensitivity up to approximately $3 \mathrm{~mm}$ with an increase to $6 \mathrm{~mm}$ when spectral ratio between selected wavelengths was obtained. Preliminary Monte Carlo results provided reasonable fit to the experimental data.
\end{abstract}

Near infrared, light scattering, cardiac tissue, depth, polarization, imaging, spectroscopy.

\section{INTRODUCTION}

Optical spectroscopy has been shown to provide promising methods for minimally invasive detection of tissue biochemical and morphological changes ${ }^{1,2}$. Diffuse reflectance and absorbance point spectroscopy is widely used in medical research in part due to technical simplicity in obtaining a broad range of structure and composition information $^{1,3}$. Additionally, spectroscopy based instrumentation holds promise for real-time pathological assessment ${ }^{1,4}$. Combining capabilities from both approaches may increase comprehensive diagnosis and maximize assistive potential in the operating room. Implementing wavelengths from the near infrared (NIR) spectral band enables subsurface investigation by avoiding blood and water absorption ${ }^{1,5,6}$. Cross-polarized light scattering reveals additional changes of optical properties due to onset and progression of disease ${ }^{7-13}$.

The aim of this work is to develop optical methods for tissue depth assessment that can be translated to clinical practice. We employ NIR spectral imaging and point spectroscopy techniques in order to test limits of real-time detection of the change in light scattering intensity as a function of tissue depth. An ex vivo porcine cardiac tissue model provided sample uniformity for proof of principle evaluation. The experimental results were modeled based on the random walk theory of photon migration ${ }^{14}$. Understanding and quantifying the light scattering signal as a function of tissue thickness is a key parameter in the development of optical techniques for diagnosis, staging, and assessment of progression of disease.

\section{EXPERIMENTAL MATERIALS AND METHODS}

Fresh porcine cardiac tissue was obtained immediately after slaughter from the UC Davis Meat Laboratory (Davis, CA). Intact heart was rinsed in tap water to remove excess blood. The heart was sliced to produce samples with homogeneous surface areas avoiding irregularities such as vascular structures. This maximized sample uniformity allowing the tissue thickness to be the controlled variable. The thickness of each sample was measured with a digital micrometer and placed individually in chilled phosphate buffered saline (PBS) solution for preservation during experimentation. Sample size varied but were each large enough to provide light scattering signal free from edge effects when detected from the center of the tissue. For image and spectra acquisition, each sample was pat dry on a kim wipe to remove excess blood and PBS solution.

\subsection{Imaging experiment}

A white light source passed through a filter wheel containing a $40 \mathrm{~nm}$ bandwidth interference filter centered at 700,850 and, $1000 \mathrm{~nm}$. The light was coupled into four fibers that uniformly illuminated the surface of the sample. Each fiber was tipped with sheet polarizers to provide linear polarized illumination. Two light scattering images, parallel and perpendicular, were acquired by rotating an additional polarizer, shown as P' in Fig. 1(a) below, for a total of 2 
polarization-sensitive NIR light scattering images per sample. Exposure time of 0.2 seconds was experimentally determined to maximize image intensity without saturation of the liquid nitrogen cooled charge coupled device (Princeton Instruments, Inc., Trenton, NJ).

Image processing code was written in Matlab, (MathWorks, Natick, MA). Background noise was subtracted from the light scattering images of each tissue sample before averaging. The average intensity was normalized against an image of a WS-1 Diffuse Reflectance Standard (Ocean Optics, Dunedin, FL). This diffusing material has a reflective outer diameter of $32 \mathrm{~mm}$ and produces more than $98 \%$ reflection from $250 \mathrm{~nm}-1500 \mathrm{~nm}$ across the surface. To normalize the raw data, the sum of the intensities of the parallel and perpendicular image components was calculated in order to obtain the total scattering intensity of the reference standard sample. The average image intensity from each tissue sample was divided by the total scattering intensity of the reference sample at the corresponding imaging wavelength. This provided an accurate estimate of the amount of backscattered light from each sample as a function of its thickness. The experimental results were predicted by a random walk theory of photon migration. Optical coefficients for this tissue model can subsequently be extracted.
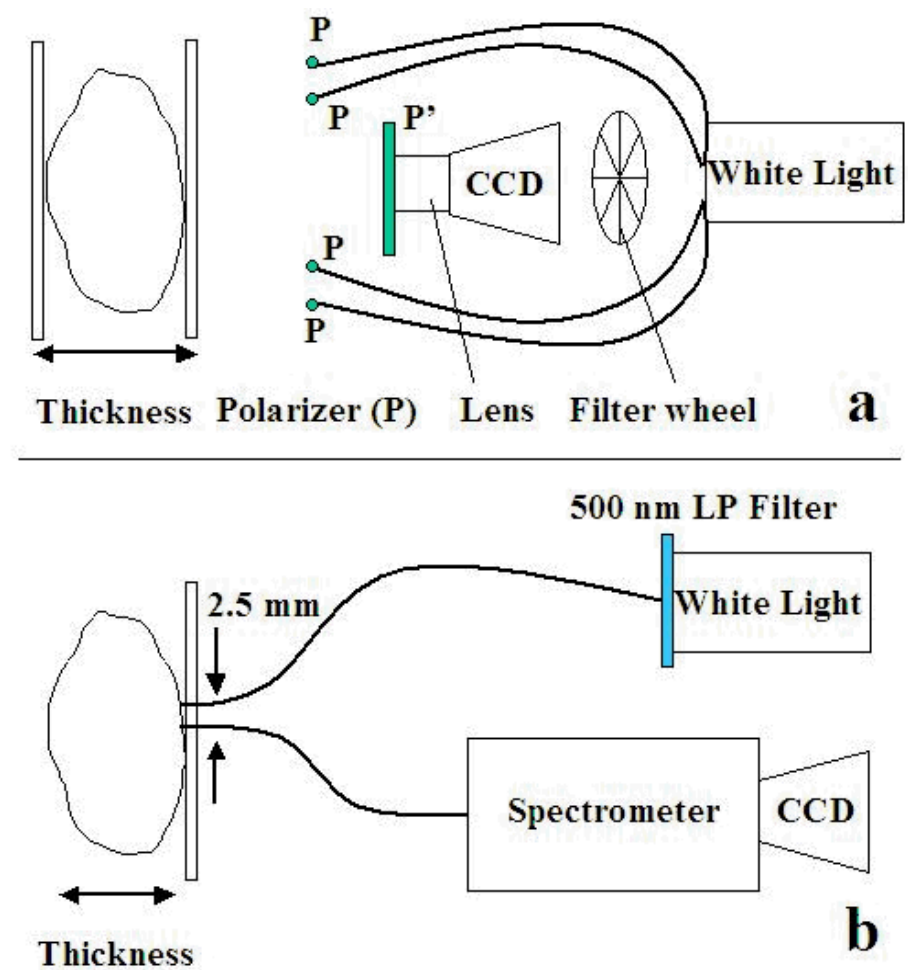

Fig. 1: System schematic of a) imaging set-up and b) spectroscopy set-up.

\subsection{Spectroscopy experiment}

A Mikropack DH-2000 halogen source (Ocean Optics, Dunedin, FL) passed through a $500 \mathrm{~nm}$ long pass filter, shown in Fig. 1(b). The filtered light was transmitted via a $600 \mu \mathrm{m}$ diameter illumination fiber that was in contact with the surface of the tissue sample on its output which was attached on the sample holder. The illumination fiber was separated by 2.5 $\mathrm{mm}$ center to center from a $250 \mu \mathrm{m}$ diameter collection fiber that was attached on the sample holder in the same manner as the illumination fiber. The collection fiber delivered the transmitted signal to a Triax Series 320 spectrometer (Jobin Yvon Inc., Edison, NJ) before detection at a liquid nitrogen cooled CCD (Princeton Instruments, Inc., Trenton, NJ). The central region of the tissue was used for each spectrum to avoid tissue thickness variability and edge effects in photon propagation. Four spectra were acquired and averaged per tissue sample to produce one average spectrum per tissue sample. The average spectra of four consecutive tissue samples were subsequently binned to produce one data point for analysis. Acquired spectra were normalized in a similar manner described in the imaging experiments (section 2.1) subtracting a dark noise spectrum and dividing against the WS-1 Diffuse Reflectance Standard spectrum. 


\section{EXPERIMENTAL RESULTS}

For comparison, the experimental results obtained at $1000 \mathrm{~nm}$ are shown in this section for all system and data analysis configurations. Results at this wavelength are representative of behaviors observed at shorter wavelengths of the NIR spectrum.

\subsection{Imaging experiment}

The average intensity of the backscattered polarized image components at $1000 \mathrm{~nm}$ is shown in Fig. 2. The normalized intensity is seen to increase for both parallel and perpendicular components before reaching a plateau at approximately 2 mm tissue thickness.

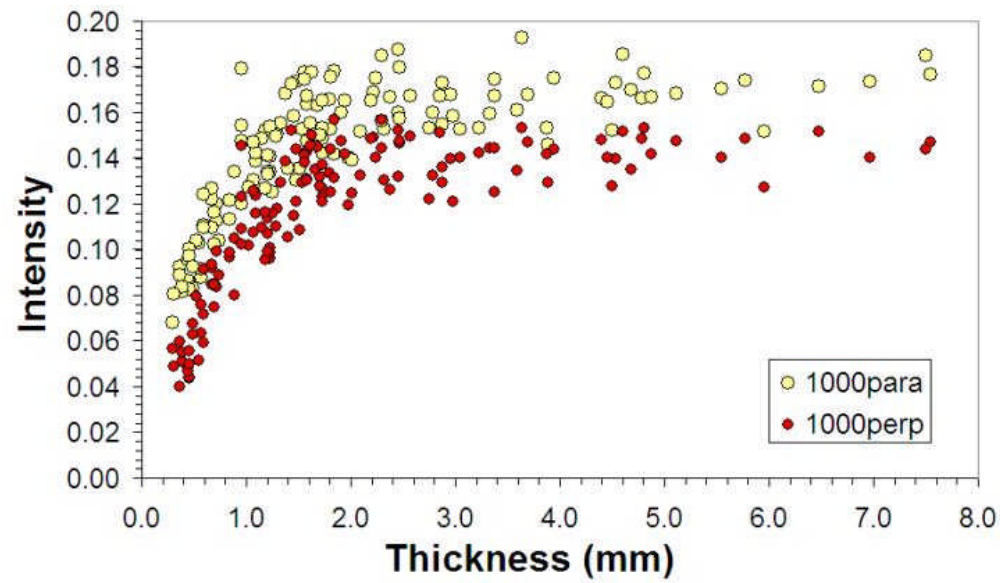

Fig. 2: Average backscatter intensities of the polarized image components at $1000 \mathrm{~nm}$ as a function of tissue thickness.

The results were analyzed using a mathematical representation of photon migration based on a random walk theory ${ }^{15}$. It should be noted that the mathematical model combined the total intensity as the sum of the polarization components minus the value attributed to specular reflection, $\left(\mathrm{I}_{/ /}+\mathrm{I}_{\perp}-0.03\right)$. This constant was determined from the model. Fig. 3 demonstrates the predictive fit to the experimental data.

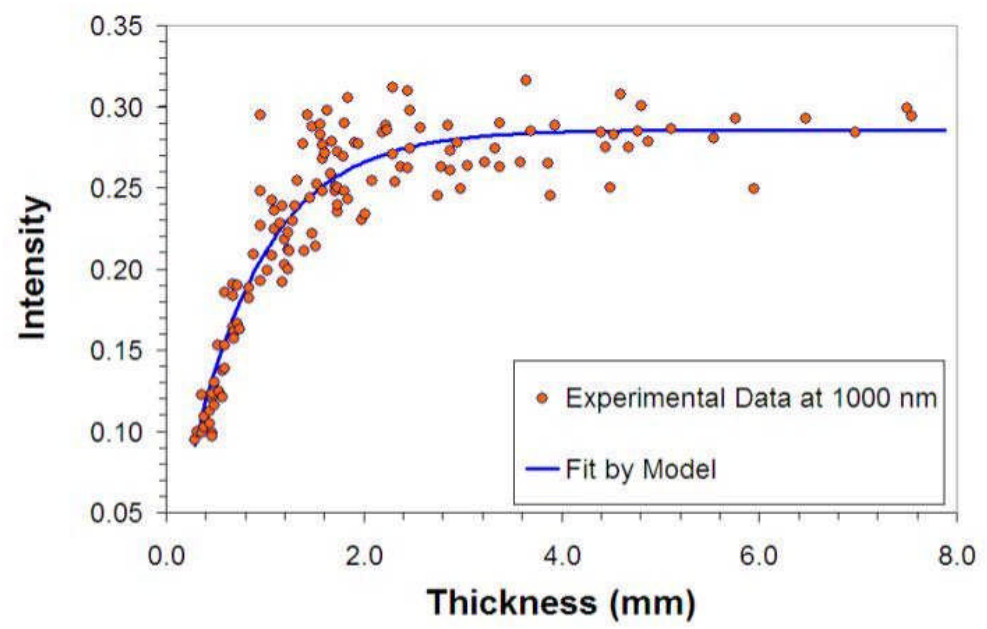

Fig. 3: Comparison between mathematical model as estimated from experimental data $\left(\mathrm{I}_{/ /}+\mathrm{I}_{\perp}-0.03\right)$ at $1000 \mathrm{~nm}$.

Optical coefficients were estimated from this model, shown in Table 1. Our results are seen to be approximately $1.5-2$ times larger than the published values in Table 2 for this tissue model. 
Table 1. The value of the optical parameters as estimated using our modeling approach

\begin{tabular}{llll}
\hline$\lambda(\mathrm{nm})$ & 700 & 850 & 1000 \\
\hline$\mu$ & 0.149 & 0.058 & 0.187 \\
$\mu_{\mathrm{at}}\left(\mathrm{mm}^{-1}\right)$ & 1.590 & 1.270 & 1.330 \\
$\mu_{\mathrm{s}}{ }^{\prime}\left(\mathrm{mm}^{-1}\right)$ & 1.380 & 1.200 & 1.120 \\
$\mu_{\mathrm{a}}\left(\mathrm{mm}^{-1}\right)$ & 0.206 & 0.070 & 0.210 \\
$\mu_{\mathrm{s}}{ }^{\prime} \mu_{\mathrm{a}}\left(\mathrm{mm}^{-2}\right)$ & 0.290 & 0.084 & 0.230 \\
\hline
\end{tabular}

Table 2. Optical Coefficients, reported in the literature

\begin{tabular}{rccccc}
\hline$\lambda(\mathrm{nm})$ & $669^{\mathrm{a}}$ & $700^{\mathrm{b}}$ & $850^{\mathrm{b}}$ & $900^{\mathrm{b}}$ & $1064^{\mathrm{a}}$ \\
\hline$\mu_{\mathrm{s}}{ }^{\prime}\left(\mathrm{mm}^{-1}\right)$ & 0.87 & 0.90 & 0.70 & 0.66 & 0.64 \\
$\mu_{\mathrm{a}}\left(\mathrm{mm}^{-1}\right)$ & 0.1 & 0.09 & 0.065 & 0.065 & 0.03 \\
\hline
\end{tabular}

${ }^{a}$ Ramshesh et al., 2003 and references therein

${ }^{\mathrm{b}}$ Swartling et al., 2003

To extract polarization information and normalize the value of the ratio with respect to the absolute intensity of the reflected light, the degree of polarization (DOP) was calculated for each sample. DOP is given as $\left(I_{/ /}-I_{\perp}\right) /\left(I_{/ /}+I_{\perp}\right)^{12}$ where $I_{/ /}$is the normalized parallel intensity and $I_{\perp}$ is the normalized perpendicular intensity. Fig. 4 illustrates the result of this calculation. Tissue depth sensitivity was observed up to approximately $1 \mathrm{~mm}$.

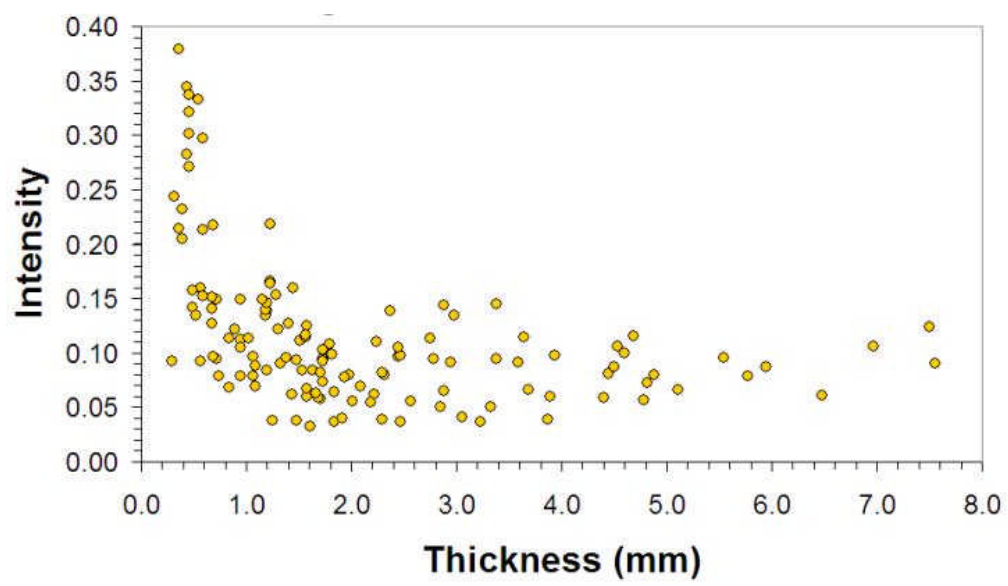

Fig. 4: Calculation of DOP as a function of tissue thickness from the $1000 \mathrm{~nm}$ image polarization components.

\subsection{Spectroscopy experiment}

The light scattering spectral response at $1000 \mathrm{~nm}$ is shown in Fig. 5. The normalized intensity is seen to increase before reaching a plateau at approximately $3 \mathrm{~mm}$. To take advantage of the spectral information rather than only the absolute intensity of the reflected light, ratios were calculated between wavelengths that offer the largest difference in photon penetration depths (see table 1). For example, Fig. 6 illustrates the light scattering response of the intensity ratio $700 \mathrm{~nm}$ $/ 1000 \mathrm{~nm}$. An increase of sensitivity to tissue depth was observed up to approximately $6 \mathrm{~mm}$. For comparison, the same analysis was performed in the imaging experiments discussed in sections 2.1nad 3.1. The results were similar as demonstrated in Fig. 7. 


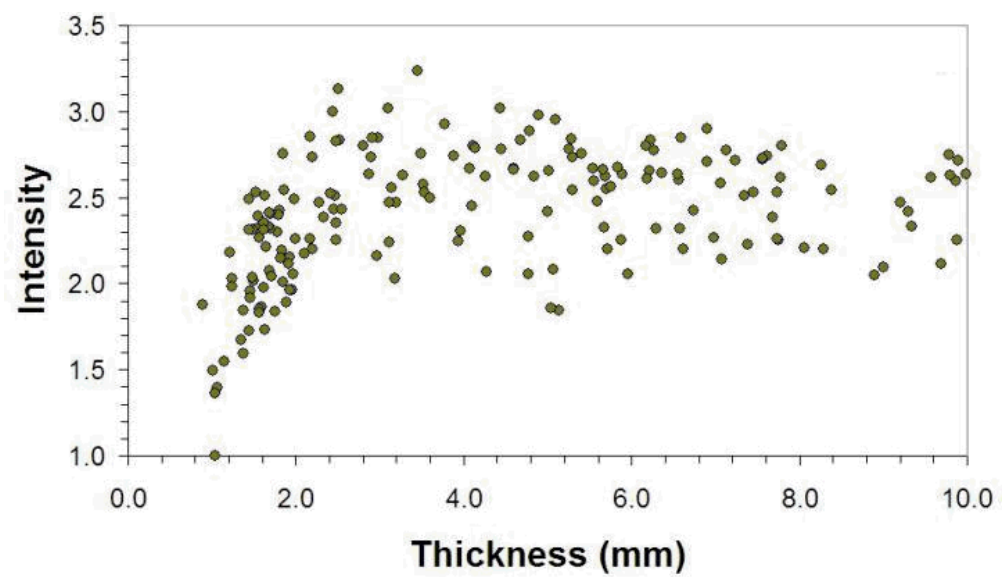

Fig. 5: Average normalized backscatter intensity at $1000 \mathrm{~nm}$ as a function of tissue thickness.

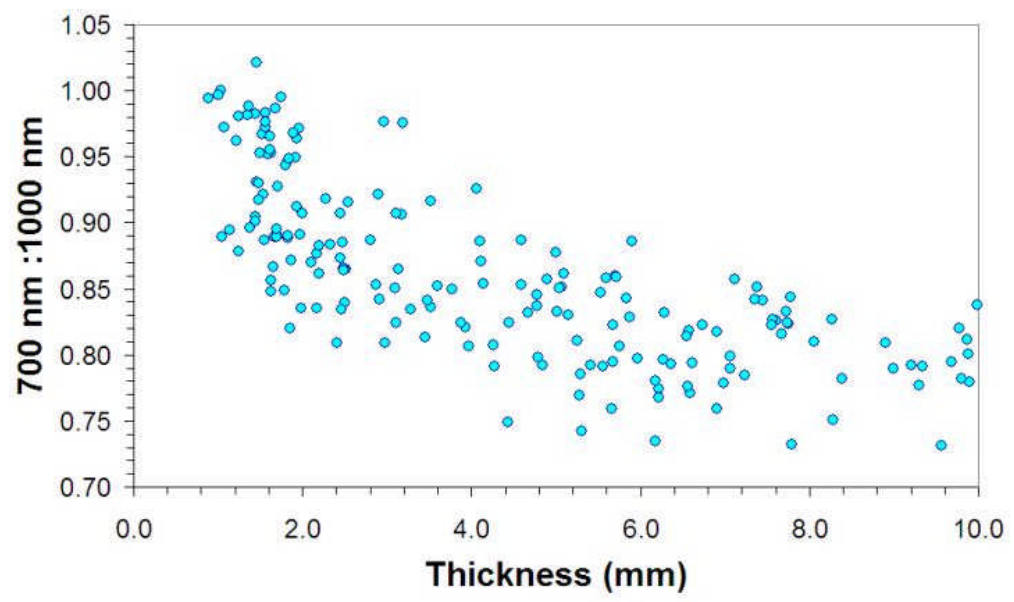

Fig. 6: The dependence of the intensity ratio at $700 \mathrm{~nm} / 1000 \mathrm{~nm}$ to tissue thickness in the dual fiber experimental configuration.

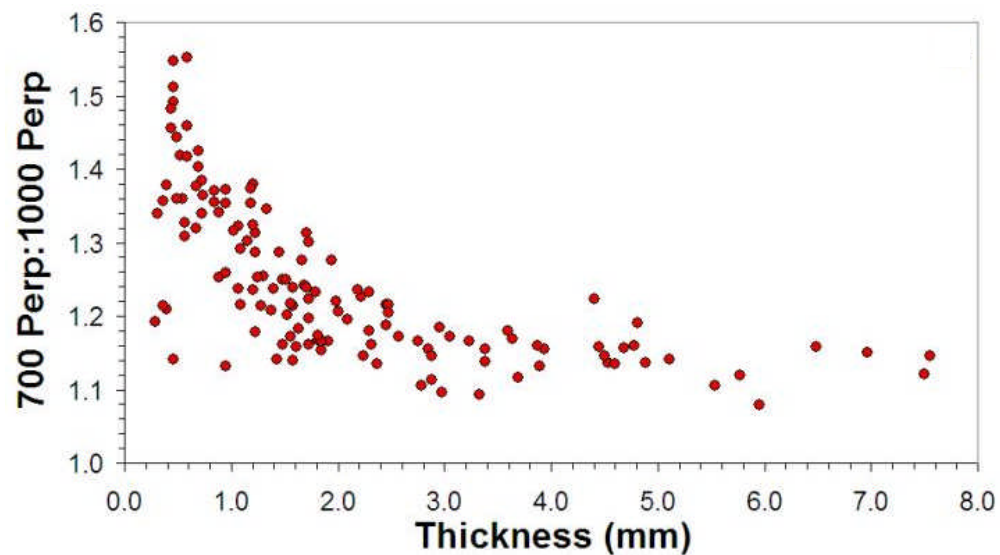

Fig. 7: The dependence of the average intensity ratio at $700 \mathrm{~nm} / 1000 \mathrm{~nm}$ to tissue thickness in the imaging experimental configuration. 


\section{DISCUSSION}

Using the imaging arrangement, the intensity in both, the parallel and the perpendicular images shown in Fig. 2 suggest a signal dependence on tissue thickness up to approximately $2 \mathrm{~mm}$. The experimental results were reasonably approximated using a mathematical interpretation based on a modified random walk theory (see Fig. 3$)^{15}$. The estimates of optical coefficients extracted from our model are nearly twice as large as literature values. This discrepancy is under further investigation but may be attributed to tissue preparation, storage, handling and, inhomogeneity of tissue composition.

The spectroscopy results shown in Fig. 5 demonstrate similar behavior as that observed in the imaging results (Fig. 2), exhibiting an increased dependence on tissue thickness. However, this increase is observed up to approximately $2 \mathrm{~mm}$ thickness in the imaging arrangement and up to approximately $3 \mathrm{~mm}$ in the dual-fiber spectroscopy arrangement. Ratio analysis of the intensity from the same tissue samples at different spectral bands uses the spectral information as a method to normalize for variations in absolute scattering intensity between tissue samples. We hypothesized that this may provide increased sensitivity to tissue thickness. The representative result of the intensity ratio at $700 \mathrm{~nm}$ over 1000 $\mathrm{nm}$ from the dual fiber experimental configuration shown in Fig. 6 and the corresponding from the imaging arrangement shown in Fig. 7 confirmed this hypothesis demonstrating an increased depth sensitivity to approximately $6 \mathrm{~mm}$ and 3 $\mathrm{mm}$, respectively.

These experimental results suggest that the reflectance spectral ratio through spatially separated fibers offers the optimal approach to evaluate tissue depth. These methods may be useful for the evaluation of tissue thickness of internal organs such as those in the gastrointestinal tract. For example, adapting fiber optic components into various types of catheters or endoscopes may incorporate these methods to clinical practice. Furthermore, the results highlight the importance of taking into account the lesion thickness in light scattering spectral imaging measurements, such as these required for functional imaging, especially if the lesion is thinner than $2 \mathrm{~mm}$. That is because the reflected signal does not depend only on the optical properties of the lesion but also on its thickness.

\section{ACKNOWLEDGMENTS}

This work performed in part under the auspices of the U.S. Department of Energy by Lawrence Livermore National Laboratory under Contract DE-AC52-07NA27344. This research is supported by funding from the Center for Biophotonics, an NSF Science and Technology Center, managed by the University of California, Davis, under Cooperative Agreement No. PHY 0120999.

\section{REFERENCES}

[1] Richards-Kortum, R., "Quantitative Optical Spectroscopy for Tissue Diagnosis," Annu. Rev. Phys. Chem., 47, 555$606(1996)$

[2] Ramanujam, N. "Fluorescence spectroscopy of neoplastic and non-neoplastic tissues." Neoplasia 2, 1-2 (2000)

[3] Alfano, R. R., Tata, D., Cordero, J., Tomashefsky, P., Longo, F., Alfano, M., "Laser-induced fluorescence spectroscopy from native cancerous and normal tissue," IEEE J. Quantum Electron 20, 1507-1511 (1984)

[4] Wagnieres, G. A., Star, W. M., Wilson, B. C., "In vivo fluorescence spectroscopy and imaging for oncological applications," Photochem. Photobiol. 68, 603-632 (1998)

[5] Lakowicz, J.R., [Principles of Fluorescence Spectroscopy] Plenum, New York, (1985)

[6] Wang, L.V., [Biomedical Optics, Principles and Imaging] John Wiley \& Sons, Inc., Hoboken, New Jersey (2007)

[7] Mourant, J.R., Bigio, I.J., Boyer, J., Conn, R.L., Johnson, T., Shimada, T., "Spectroscopic diagnosis of bladder cancer with elastic light scattering," Lasers Surg. Med. 17, 350-357 (1995)

[8] Kim, Y.L., Liu, Y., Wali, R.K., Roy, H.K., Goldberg, M.J., Kromin, A.K., Chen, K., Backman, V., "Simultaneous measurement of angular and spectral properties of light scattering for characterization of tissue microarchitecture and its alteration in early precancer," IEEE J. Sel. Top. Quantum Electron 9, 243-256 ( 2003)

[9] Drezek, R., Guillaud, M., Collier, T., Boiko, I., Malpica, A., Macaulay, C., Follen, M., Richards-Kortum, R., "Light scattering from cervical cells throughout neoplastic progression: influence of nuclear morphology, DNA content, and chromatin texture," J. Biomed. Opt. 8, 7-16 (2003)

[10] Zonios, G., Perelman, L. T., Backman, V. M., Manoharan, R., Fitzmaurice, M., Van Dam, J., Feld, M. S., "Diffuse reflectance spectroscopy of human adenomatous colon polyps in vivo," Appl. Opt. 38 (31), 6628-6637 (1999) 
[11] Mourant, J. R., Freyer, J. P., Hielscher, A. H., Eick, A. A., Shen, D., Johnson , T. M., "Mechanisms of light scattering from biological cells relevant to noninvasive optical-tissue diagnostics," Appl. Opt. 37, 3586-3593 (1998)

[12] Demos, S. G., Alfano, R. R., "Optical polarization imaging," Appl. Opt. 36, 150-155 (1997)

[13] Demos, S. G., Radousky, H. B., Alfano, R. R., "Deep subsurface imaging in tissues using spectral and polarization filtering," Opt Express 7, 23-28 (2000)

[14] Weiss, G. H., Gandjbakhche, A. H., Masoliver, J., "Isotropization Length for Random-Walk Models of Photon Migration in Turbid Media," J. Mod. Opt. 42,1567-1574 (1995)

[15] Lin, B., Chernomordik, V., Gandjbakhche, A. H., Matthews, D. L., Demos, S. G., "Investigation of signal dependence on tissue thickness in near infrared spectral imaging," Opt Express 15, 25 (2007) 